\title{
The SUMOylation Pathway as a Potential Therapeutic Target in Multiple Myeloma
}

\author{
James J. Driscoll \\ Division of Hematology and Oncology, Department of Internal Medicine \\ University of Cincinnati Medical Center and Barrett Cancer Center \\ Cincinnati, $\mathrm{OH}$ \\ Medical Oncology Branch, National Cancer Institute, National Institutes of Health \\ Bethesda, $M D$ \\ USA
}

\section{Introduction}

The Ubiquitin+Proteasome System (UPS) represents a successful anti-cancer strategy that has translated from preclinical studies to clinical development and significantly improved the survival of Multiple Myeloma (MM) patients (1). The proteasome is high molecular weight structure that contains multiple proteolytic sites and functions as the catalytic core of the UPS to degrade Ub conjugated proteins (2-4). Inhibition of the proteasome's chymotryptic-like peptidase activity blocks the entire UPS and leads to selective tumor cell death. Bortezomib is the first proteasome inhibitor used in human clinical trials and treatment leads to a reduction in tumor burden in MM patients $(5,6)$. However, cellular resistance to proteasome-based therapy generally results and reduces the efficacy. Resistance to proteasome inhibitors may result from altered expression of either proteasome or non-proteasome encoding genes. SUMOylation is a post-translational modification that attaches a Small Ubiquitin-like MOdifier to target proteins and, similar to Ub, may target proteins for proteasomal degradation. Gene expression profiling of MM patient samples indicated that bortezomib resistance may be achieved through induction of non-proteasome components. Importantly, certain effectors of the SUMOylation pathway were induced in the samples of MM patients that did not respond to bortezomib. A molecular-based, biologically-relevant supervised approach was used to identify a compendium of genes within the SUMO+UPS that were induced in MM patients and correlated with a decreased response to bortezomib. This molecular approach is relevant to a heterogeneous disease like MM where patients with similar clinical and pathologic features have vastly different genetic mutations responsible for disease and varying responses to therapies and clinical outcomes. The potential role of the SUMOylation pathway is only beginning to emerge, however, evidence indicates that SUMOylation is induced in myelomagenesis and also in bortezomib-resistant cells.

An aggresome is a proteinaceous inclusion body that forms when the cellular protein degradation machinery is impaired or overwhelmed and leads to an accumulation of proteins for disposal $(7,8)$. Aggresome formation is thought to represent a cellular protective 
response to the presence of abnormal, misfolded or damaged proteins within the cytoplasm that cannot be eliminated by the UPS. Aggresomes form adjacent to the microtubuleorganizing center (MTOC) and neighboring the centrosome. The microtubule-based motor protein dynein, heat shock proteins and the histone deacetylase 6 (HDAC6) have been reported as aggresome components (9). HDAC6 specifically functions as an adaptor protein between the dynein motor protein and accumulating polyubiquitinated substrate proteins. The protein aggregates that reside in an aggresome are sequestered in a cage that consists largely of intermediate filaments. Aggresome formation may serve as an alternative mechanism to eliminate the cytotoxic effect of accumulated $\mathrm{Ub} \sim$ protein conjugates. Recent evidence indicates that SUMOylation effectors and SUMO-modified proteins are related to aggresome formation. Inhibition of SUMOylation could preclude aggresome formation and consequently overcome bortezomib resistance.

The formation of the aggresome functions as a mechanism not only to sequester potentially cytotoxic aggregates but also to serve as a platform for the eventual autophagic clearance of these aggregates $(10,11)$. Autophagy is a catabolic process that involves the degradation of the cell's own constituents through the lysosomal machinery $(12,13)$. It is a tightly-regulated process that plays a normal part in cell growth, development, and homeostasis and maintains the balance between the synthesis, degradation and subsequent recycling of cellular products. It is a major mechanism by which a starving cell reallocates nutrients from unnecessary processes to more-essential processes. A variety of autophagic processes exist, all having in common the degradation of intracellular components through the lysosome. The most well-known mechanism of autophagy involves the formation of a membrane around a targeted region of the cell, separating the contents from the rest of the cytoplasm. The resultant vesicle then fuses with a lysosome and subsequently degrades the contents.

Importantly, new evidence indicates upon proteasome inhibition, the accumulated $\mathrm{Ub} \sim$ protein conjugates are bound by autophagy cargo receptors for clearance $(14,15)$. Thus, it appears that aggresome formation is a prerequisite for autophagic clearance of $\mathrm{Ub} \sim$ protein conjugates (16). In addition, selective autophagy may serve to eliminate these aggregates and overcome proteasome inhibition and yield drug resistance. It remains to be determined whether SUMOylated proteins are also cleared by autophagic clearance. However, inhibition of the SUMOylation pathwya may prevent aggresome formation, impede their autophagic clearance of $\mathrm{Ub} \sim$ protein conjugates and overcome bortezomib resistance. Future studies will address these questions and may yield new therapeutic targets, novel anti-cancer agents and lead to improved strategies for the treatment of MM and other malignancies.

\section{Clinical success of proteasome inhibitor-based therapy in multiple myeloma}

Multiple myeloma (MM) is a hematologic neoplasm of B-cell origin characterized by the clonal proliferation and progressive accumulation of malignant plasma cells within the bone marrow. MM accounts for greater than $10 \%$ of all hematologic cancers and in spite of conventional high-dose chemotherapies, remains uniformly fatal, in part, because of either intrinsic (primary) or acquired (secondary) drug resistance. Recent advances in translational medicine have led to the development of molecularly-based targeted therapies as well as 
highly effective synergistic drug combinations that have the potential to achieve a higher frequency of durable responses in a greater number of MM patients. Molecular, genomic and proteomic studies have further increased understanding of the biology of myelomagenesis, provided the basis for better prognostic classification in the context of uniform therapies and generated a sound rationale for combining targeted therapies in current and emerging clinical development.

The $26 \mathrm{~S}$ proteasome is a high-molecular weight, multi-subunit complex that consists of a $20 \mathrm{~S}$ catalytic core and a 195 regulatory particle. Proteasomes are found in eukaryotes, archaebacteria and some bacteria. The $20 \mathrm{~S}$ proteasome functions as the catalytic core of the ubiquitin-proteasome system to hydrolyze proteins into short polypeptide and is capable of hydrolyzing peptides C-terminal to chymotryptic, tryptic or acidic residues. 20S proteasomes are cylindrical structures composed of four stacked rings with each ring composed of seven individual subunits. The two inner rings are made of seven $\beta$ subunits that contain the various active sites and the two outer rings are each composed of seven $a$ subunits. The two outer rings comprised of a subunits are then bound at either pole by the 195 regulatory particles.

Proteasome inhibition is a promising therapeutic strategy for the treatment of an increasing number of B cell malignancies, especially MM. Bortezomib is a low molecular weight boronbased agent that inhibits the chymotryptic-like activity of the proteasome. Inhibition of the chymotryptic-like activity inhibits all proteasome activity and acts as a blockade of the entire ubiquitin+proteasome pathway. The result is the intracellular accumulation of $\mathrm{Ub} \sim$ protein conjugates, activation of cellular stress sensors and eventually cell death. The selective cytotoxic effect of proteasome inhibition on tumor cells further validated protein degradation as a therapeutic target in oncology and has been translated in the treatment of certain malignancies. Bortezomib was US Food and Drug Administration (FDA) approved for the treatment of newly diagnosed, relapsed and refractory MM. The introduction of bortezomib into the treatment of $\mathrm{MM}$ resulted in a paradigm shift and significantly improved patient survival. The concurrent targeting of both the tumor cell as well as the surrounding bone marrow microenvironment may promote tumor cell death and efficacy. The FDA has recently approved a more effective three drug combination of bortezomib, lenalidomide and dexamethasone for the treatment of MM that may act on the malignant plasma cell and also to counteract the protective effect of the bone marrow milieu.

Many MM patients, however, exhibit an intrinsic (primary) resistance and do not initially respond to bortezomib. Furthermore, in those patients that do respond, clinical efficacy is dampened by the inevitable emergence of acquired drug-resistance that eventually develops through unidentified mechanisms. While bortezomib has significantly transformed the management of $\mathrm{MM}$, the mechanism(s) of action and bases of individual patient susceptibility remain unclear. These recent advances in mechanistic understanding and treatment modalities have extended median survival to $>6$ years and $10 \%$ of patients now survive $>10$ years. However, the vast majority of MM remains incurable even with these strategies.

Though the catabolism of ubiquitinated substrates has been targeted therapeutically with significantly improved prognosis, patient response to bortezomib remains highly variable and cannot be predicted accurately. E3 Ub ligases are the specific components of the UPS 
that recognize substrates for degradation and confer specificity on target selection for the UPS. Therefore, the expression of individual E3's was analyzed using a microarray dataset obtained from MM patient tumor samples and found a striking variability in the expression level of individual E3 Ub ligases between normal plasma cells and patients MM cells. Specifically, RNF4, an E3 specific for poly-sumoylated proteins, was induced in MM patients and correlated with decreased patient response to the proteasome inhibitor bortezomib. Expression profiling of pretreatment tumor samples obtained from MM patients in independent clinical trials were used to generate a signature that correlated expression of $\mathrm{SUMO}+\mathrm{Ub}+$ Proteasome pathway components with clinical outcome to predict patient response to bortezomib.

\section{The Ub+Proteasome System (UPS) and the Ub-like SUMOylation pathway}

The UPS regulates numerous critical cellular processes by maintaining the appropriate intracellular level of key proteins. Whereas de novo protein synthesis is a comparatively slow process, proteins are rapidly degraded at a rate compatible with the control of cell cycle transitions and cell death induction. The critical role played by Ub-mediated protein turnover in cell cycle regulation makes this process a high-value target for oncogenic mutations. SUMO proteins are similar to ubiquitin, and SUMOylation is directed by an three-step enzymatic cascade analogous to that involved in ubiquitination. SUMOylation is a highly dynamic, reversible, post-translational protein modification, similar to ubiquitination that attaches a family of polypeptides to target proteins to modify their function, subcellular localization or stability. A role for SUMOylation in the UPS has been established since SUMO attachment signals the recruitment of specific E3 Ub ligases to target proteins, e.g., PML, PEA, PARP-1 and HIF1-alpha, for ubiquitination and proteasomal degradation. Induction of individual SUMOylation and UPS effectors has been demonstrated in MM cell lines and patient samples and SUMO pathway induction has been correlated with resistance to bortezomib-based therapy. Similarly, proteasome components are induced in bortezomib-resistant cells ad ndecrease the toxic accumulation of $\mathrm{Ub} \sim$ conjugates. The mechanistic link of SUMOylation to proteasomal resistance is uncertain. An important unresolved issue is the clinical significance of sumoylation during proteasomal degradation and proteasome inhibitor-based therapy. SUMOylation was recently shown to have a role in proteasomal degradation since SUMO-2/3 attachment can signal Ub-dependent degradation. Novel RING family Ub ligases, e.g., RNF4, bear SUMO interaction motifs and have been implicated in the proteasomal degradation of SUMOmodified target protein PML. Similarly, the E3 Ub ligase von Hippel-Lindau (VHL) has been reported to control the levels of sumoylated HIF-1a while VHL and RNF4 control the levels of HIF-2 $\square$ to indicate that sumoylated HIF-2a is degraded through SUMO-targeted ubiquitination. SUMO modification as a signal for degradation is conserved in eukaryotes and $\mathrm{Ub}$ ligases that specifically recognize SUMO-modified proteins have been discovered from yeasts to humans. proteasome components to further link the pathways. Individual components of the sumoylation pathway serve as biomarkers to predict clinical response to bortezomib and provide evidence for targeting SUMO pathway to improve therapeutic outcome in myeloma in general and specifically in combination with proteasome inhibitors.

SUMO modification as a signal for degradation is conserved in eukaryotes and Ub ligases that specifically recognize SUMO-modified proteins have been discovered from yeasts to 
humans. E3 Ub ligases are engaged in the recognition of biologically relevant proteins that are degraded by the proteasome. While proteasome inhibition generates the accumulation of $\mathrm{Ub} \sim$ conjugated proteins and ultimately cell death, the selectivity of this process and the role of individual E3 ligases has not been previously addressed. We investigated whether UPS effectors and specifically, E3 ligases were differentially expressed in MM patients and whether expression correlated with resistance to bortezomib-based therapy. Recently, we demonstrated that genes encoding SUMOylation pathway effectors were induced during myelomagenesis and correlated with decreased patient survival.

\section{Aggresomes and proteasome-based therapy}

Aggresomes are novel cellular structures that employ HDAC6 to sequester and transport protein aggregates to the autophagy-lysosome system for efficient disposal. These poorly defined structures have been shown to consist of HDAC6, microtubules, heat shock proteins and ubiquitin. Aggresome formation is induced by a number of cellular and genotoxic stressors, including proteasomal inhibition. These structures may represent a potential compensatory mechanism to for cells to eliminate $\mathrm{Ub} \sim$ protein conjugates that accumulate upon proteasomal inhibiton. The result may be resistance to proteasome inhibitors and promote tumor survival. Similar to $\mathrm{Ub}$, the small Ub-like MOdifier (SUMO) is attached to target certain proteins for proteasomal degradation but an association with aggresomes has not been established. The proteasome is limited in its capacity to degrade certain proteins, such as membrane-associated, oligomeric and protein aggregates. SUMOylation, like ubiquitination, may be mechanistically and biologically linked to aggresomes to promote the removal of toxic proteins that accumulate upon proteasomal inhibition. SUMOylation may also be required for the formation of aggresomes. Genetic or pharmacologic targeting of SUMOylation pathway effectors may impair functioning and formation of aggresomes to overcome drug resistance and enhance the cytotoxicity of proteasome inhibitors.

\section{Autophagy}

Removal of harmful protein aggregates is mediated by autophagy, a mechanism that sequesters cytosolic cargo and delivers it to the lysosome (17-20). Autophagy is of great importance for cellular homeostasis and survival, while its deregulation has been linked to pathological conditions, e.g., certain neurodegenerative diseases and cancer (21-23). The process of autophagy for many years has been thought of as a random degradative process to eliminate long-lived cell constituents. However, recent evidence indicates that autophagy can be selective and mediated through specialized Ub-binding cargo receptors.

The involvement of $\mathrm{Ub}$ as a specificity factor for selective autophagy has resulted in a rapid emergence of studies that have demonstrated active communication between the UPS and the aggresome-autophagy-lysosomal system (25). The identification and characterization of autophagy receptors, such as p62/SQSTM1, NBR1, p97/ VCP which can simultaneously bind both $\mathrm{Ub}$ and autophagy-specific Ub-like modifiers, e.g., LC3/GABARAP, and possible SUMO has provided a molecular link between the UPS and autophagy (26-28). Since the UPS regulates the selective degradation of certain short-lived, mutant and misfolded proteins, autophagy may remove not only aggregates which are comprised of long-lived proteins but also UPS substrates. Selective autophagy may protect cells from oxidative and 
genotoxic stresses. In addition, this process may remove $\mathrm{Ub} \sim$ and $\mathrm{SUMO} \sim$ protein conjugates that accumulate upon proteasome inhibition and overcome the cytotoxic effect of bortezomib. Induction of selective autophagy may be induced in tumor cells to provide a mechanism of cell survival and drug resistance $(29,30)$. Selective autophagy represents a promising new pathway to investigate as means to overcome resistance to proteasome inhibitors and further advance the treatment of MM.

\section{Conclusions}

Multiple myeloma accounts for a substantial amount of all hematologic malignancies seen worldwide. In the US and Europe, the incidence of MM exceeds 40,000 cases annual. MM has assumed a position of prominence as a model system for preclinical studies, drug development and clinical trials. Unlike other malignancies, in which surgery and radiation are important treatment modalities, $\mathrm{MM}$ is nearly exclusively treated with stem cell transplantation (SCT) or synergistic chemotherapeutic combinations. SCT has produced statistically significant survival benefit for MM patients. However, a number of MM patients are not eligible for SCT either because of age, toxicities and many patients can relapse. Novel therapeutic agents such as the immunomodulatory agents and bortezomib have revolutionized therapy and improved overall survival. However, despite these advances, MM remains largely incurable as disease relapse is inevitable. Therefore, there is an urgent need for new, tolerable agents that demonstrate the ability to either overcome or prevent drug resistance. The role of the SUMOylation pathway in Multiple Myeloma is only beginning to be understood and appears to be a novel therapeutic target that can be used not only to treat MM but also to overcome drug resistance. Future studies will address the role of this emerging pathway in myelomagenesis.

\section{References}

[1] Hideshima T, Bradner JE, Chauhan D, Anderson KC. Intracellular protein degradation and its therapeutic implications. Clin Cancer Res 2005;11:8530-8533.

[2] Hershko, A. and Ciechanover, A. The ubiquitin system. Annu. Rev. Biochem. 1998; 67, 425-479.

[3] Kirkin V, Dikic I. Ubiquitin networks in cancer. Curr Opin Genet Dev. 2011, 21:21-28.

[4] Kerscher O, Felberbaum R, Hochstrasser M. Modification of proteins by ubiquitin and ubiquitin-like proteins. Annu Rev Cell Dev Biol. 2006;22:159-180.

[5] Hideshima T, Bradner JE, Wong J, et al. Small-molecule inhibition of proteasome and aggresome function induces synergistic antitumor activity in multiple myeloma. Proc Natl Acad Sci U S A 2005;102(24):8567-8572.

[6] Mitsiades CS, Davies FE, Laubach JP, et al. Future directions of next-generation novel therapies, combination approaches, and the development of personalized medicine in myeloma. J Clin Oncol 2011 29:1916-1923.

[7] Kopito, R R. Aggresomes, inclusion bodies and protein aggregation. Trends Cell Biol. 2000; 10:524-30.

[8] Pang, T. The Role of Ubiquitin in Autophagy-Dependent Protein Aggregate Processing Genes and Cancer 2010; 779- 786. 
[9] Kawaguchi, Y., Kovacs, J. J., McLaurin, A., Vance, J. M., Ito, A., and Yao, T.-P. The deacetylase HDAC6 regulates aggresome formation and cell viability in response to misfolded protein stress. Cell. 115. 727-738 (2003).

[10] Kirkin V, McEwan DG, Novak I, Dikic I., A Role for Ubiquitin in Selective Autophagy. Mol Cell 2010; 34:259-269.

[11] Kondo Y, Kanzawa T, Sawaya R, Kondo S. The role of autophagy in cancer development and response to therapy. Nat Rev Cancer 2005; 5: 726-34.

[12] White E., DiPaola R. S. The Double-Edged Sword of Autophagy Modulation in Cancer. Clin. Cancer Res. 2009 15, 5308-5316.

[13] 50. Mathew R, et al. Role of autophagy in cancer. Nat Rev Cancer. 2007;7:961-967.

[14] I. Dikic, T. Johansen, V. Kirkin, Selective autophagy in cancer development and therapy. Cancer Res. 70, 3431-3434 (2010).

[15] Amaravadi, R.K., Thompson, C.B. 2007. The roles of therapy-induced autophagy and necrosis in cancer treatment. Clin. Cancer Res. 13:7271-7279.

[16] Hoeller D, Dikic I. Targeting the ubiquitin system in cancer therapy. Nature; 2009; 458 (7237): 438-44.

[17] Dikic I, Johansen T, Kirkin V. Selective autophagy in cancer development and therapy. Cancer Res. 2010, 70(9):3431-4.

[18] Kraft C, Peter M, Hofmann K. Selective autophagy: ubiquitin-mediated recognition and beyond. Nat Cell Biol. 2010;12:836-841.

[19] Amaravadi RK, Lippincott-Schwartz J, Yin XM, Weiss WA, Takebe N, Timmer W, DiPaola RS, Lotze MT, White E. (2011) Principles and current strategies for targeting autophagy for cancer treatment. Clin Cancer Res. Feb 15;17(4):654-66.

[20] White, E., and DiPaola, R. S. (2009). The double-edged sword of autophagy modulation in cancer. Clinical Cancer Res. 15(17):5308-16.

[21] Levine B. and Kroemer G. Autophagy in the pathogenesis of disease. Cell 2008; 132:2742.

[22] Shintani T, Klionsky DJ. Autophagy in health and disease: a double-edged sword. Science 2004; 306: 990-5.

[23] Rubinsztein DC: The roles of intracellular protein-degradation pathways in neurodegeneration. Nature 2006, 443:780-786.

[24] Korolchuk VI, Menzies FM, Rubinsztein DC. Mechanisms of cross-talk between the ubiquitin-proteasome and autophagy-lysosome systems. FEBS Lett 584: 1393-1398, 2010.

[25] Lee, J.-Y. and T.-P. Yao (2010). "Quality control autophagy: A joint effort of ubiquitin, protein deacetylase and actin cytoskeleton." Autophagy 6(4): 555-557.

[26] Xie Z, Klionsky DJ. Autophagosome formation: core machinery and adaptations. Nat Cell Biol. 2007;9:1102-1109.

[27] Komatsu M, Waguri S, Koike M, et al. Homeostatic levels of p62 control cytoplasmic inclusion body formation in autophagy-deficient mice. Cell 2007;131:1149-63.

[28] 35. Kirkin V, Lamark T, Sou Y, Bjorkoy G, Nunn J, Bruun J, Shvets E, Mc Ewan D, Clausen T, Wild P, Bilusic I, Theurillat JP, Overvatn A, Ishii T, Elazar Z, Komatsu M, Dikic I, Johansen T (2009) A role for NBR1 in autophagosomal degradation of ubiquitinated substrates. Mol Cell 33:505-516

[29] Hoang B, Benavides A, Shi Y, Frost P, Lichtenstein A. Effect of autophagy on multiple myeloma cell viability. Mol Cancer Ther2009;8:1974-84. 
[30] Catley L, Weisberg E, Kiziltepe T, et al.(2006) Aggresome induction by proteasome inhibitor bortezomib and alpha-tubulin hyperacetylation by tubulin deacetylase (TDAC) inhibitor LBH589 are synergistic in myeloma cells. Blood 2006, 108:34413449 . 


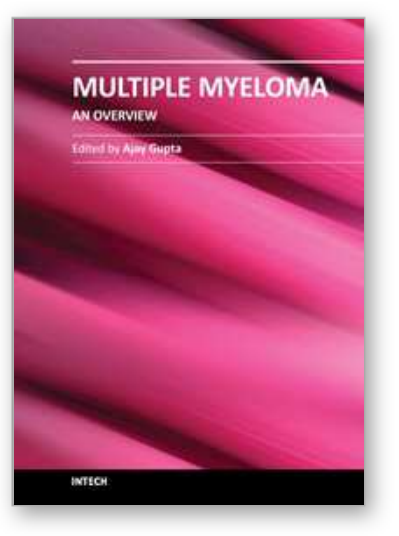

\author{
Multiple Myeloma - An Overview \\ Edited by Dr. Ajay Gupta
}

ISBN 978-953-307-768-0

Hard cover, 274 pages

Publisher InTech

Published online 20, January, 2012

Published in print edition January, 2012

Multiple myeloma is a malignant disorder characterized by the proliferation of plasma cells. Much insight has been gained into the molecular pathways that lead to myeloma and indeed much more remains to be done. The understanding of these pathways is closely linked to their therapeutic implications and is stressed upon in the initial chapters. Recently, the introduction of newer agents such as bortezomib, lenalidomide, thalidomide, liposomal doxorubicin, etc. has led to a flurry of trials aimed at testing various combinations in order to improve survival. Higher response rates observed with these agents have led to their integration into induction therapies. The role of various new therapies vis a vis transplantation has also been examined. Recent advances in the management of plasmacytomas, renal dysfunction, dentistry as well as mobilization of stem cells in the context of myeloma have also found exclusive mention. Since brevity is the soul of wit our attempt has been to present before the reader a comprehensive yet brief text on this important subject.

\title{
How to reference
}

In order to correctly reference this scholarly work, feel free to copy and paste the following:

James J. Driscoll (2012). The SUMOylation Pathway as a Potential Therapeutic Target in Multiple Myeloma, Multiple Myeloma - An Overview, Dr. Ajay Gupta (Ed.), ISBN: 978-953-307-768-0, InTech, Available from: http://www.intechopen.com/books/multiple-myeloma-an-overview/the-sumoylation-pathway-as-a-potentialtherapeutic-target-in-multiple-myeloma

\section{INTECH}

open science | open minds

\author{
InTech Europe \\ University Campus STeP Ri \\ Slavka Krautzeka 83/A \\ 51000 Rijeka, Croatia \\ Phone: +385 (51) 770447 \\ Fax: +385 (51) 686166 \\ www.intechopen.com
}

\author{
InTech China \\ Unit 405, Office Block, Hotel Equatorial Shanghai \\ No.65, Yan An Road (West), Shanghai, 200040, China \\ 中国上海市延安西路65号上海国际贵都大饭店办公楼 405 单元 \\ Phone: +86-21-62489820 \\ Fax: $+86-21-62489821$
}


(C) 2012 The Author(s). Licensee IntechOpen. This is an open access article distributed under the terms of the Creative Commons Attribution 3.0 License, which permits unrestricted use, distribution, and reproduction in any medium, provided the original work is properly cited. 